\title{
Educational Technology Network: A Computer Conferencing System Dedicated to Applications of Computers in Radiology Practice, Research, and Education
}

\author{
Michael P. D'Alessandro, Michael J. Ackerman, and Susan M. Sparks
}

\begin{abstract}
Educational Technology Network (ET Net) is a free, easy to use, on-line computer conferencing system organized and funded by the National Library of Medicine that is accessible via the SprintNet (SprintNet, Reston, VA) and Internet (Merit, Ann Arbor, MI) computer networks. It is dedicated to helping bring together, in a single continuously running electronic forum, developers and users of computer applications in the health sciences, including radiology. ET Net uses the Caucus computer conferencing software (CamberRoth, Troy, NY) running on a microcomputer. This microcomputer is located in the National Library of Medicine's Lister Hill National Center for Biomedical Communications and is directly connected to the SprintNet and the Internet networks. The advanced computer conferencing software of ET Net allows individuals who are separated in space and time to unite electronically to participate, at any time, in interactive discussions on applications of computers in radiology. A computer conferencing system such as ET Net allows radiologists to maintain contact with colleagues on a regular basis when they are not physically together. Topics of discussion on ET Net encompass all applications of computers in radiological practice, research, and education. ET Net has been in successful operation for 3 years and has a promising future aiding radiologists in the exchange of information pertaining to applications of computers in radiology.

Copyright 1993 by W.B. Saunders Company
\end{abstract}

KEY WORDS: Computer conferencing, bulletin board systems, education, radiology, computers.

$\mathbf{T}$ HE EDUCATIONAL Technology Network (ET Net) is a free, easy to use, on-line computer conferencing system organized by the National Library of Medicine that is accessible via the SprintNet (SprintNet, Reston, VA) and Internet (Merit, Ann Arbor, MI) computer networks. It is dedicated to helping bring together, in a single continuously running electronic forum, developers and users of computer applications in health sciences education and radiology.

\section{METHODS}

ET Net uses the Caucus computer conferencing software (Camber-Roth, Troy, NY) running on a Compaq 386 microcomputer (Compaq, Houston, TX) using the XENIX operating system (Santa Cruz Organization, Santa Cruz, CA). This microcomputer is located in the National Library of Medicine's Lister Hill National Center for Biomedical Communications (National Institutes of Health, Bethesda, MD) and is directly connected to Sprint Net, a commercial worldwide computer network. A user connects to ET Net by placing a phone call to their local SprintNet node using a personal computer and a modem; the SprintNet node then connects them to ET Net. ET Net is also connected to the Internet, an academic worldwide computer network. Users of the Internet may access ET Net directly. ET Net currently is configured to allow its simultaneous use by four SprintNet and 12 Internet users. As part of its mission of disseminating medical information to health science professionals, the National Library of Medicine absorbs the cost of managing ET Net, as well as the SprintNet charges for ET Net access.

ET Net is organized hierarchically and consists of several computer conferences, each encompassing a broad subject area of potential discussions. Each conference is further subdivided into items, with each item serving as a starting point for a discussion on a topic. Any conference participant may start a new item. Discussion is carried out when participants add responses directly to the appropriate item. Each conference has an index of all its items. Each time one enters an ET Net conference, you are shown the new responses to old items and the new items that have been added since your last visit. You are given the opportunity to add your own responses to preexisting items and to add your own new items.

Because ET Net was conceived to facilitate communications between health sciences professionals using computers in health sciences education, most discussions on ET Net deal with applications of computers in this area. Recent discussion topics include integration of computers into health sciences educational programs in the basic and clinical sciences; development of new and evaluations of existing health sciences educational software; uses of videodisc and CD-ROM hardware technologies in health sciences education; uses of multimedia and artificial intelligence software technologies in health sciences education;

From the Department of Radiology, Electric Differential Multimedia Laboratory, University of Iowa College of Medicine, Iowa City, IA; and the National Libran of Medicine, Lister Hill National Center for Biomedical Communications, Bethesda, MD.

Supported by a grant from the National Library of Medicine's Lister Hill National Center for Biomedical Communications, Bethesda, MD.

Address reprint requests to Michael $P$. D'Alessandro, $M D$, Department of Radiology, University of Iowa Hospitals and Clinics, 200 Hawkins Dr, Iowa City, IA 52242-1009.

Copyright $\odot 1993$ by W.B. Saunders Company

0897-1889/93/0604-0005\$03.00/0 
computing in health sciences libraries; training and careers in medical informatics; and the ultimate goal of easily organizing and disseminating medical information.

Additionally, there is a conference on ET Net, entitled "digital_images," which is dedicated to applications of computers in radiological practice, research, and education. Topics of discussion include, but are not limited to, radiological education via multimedia and computer-aided instruction systems; digital radiography and picture archiving and communication systems (PACS); image processing and three-dimensional image reconstruction techniques; teleradiology; use of high-speed computer networks in radiology; radiology information systems (RIS); uses of artificial intelligence in radiology; and how computers can aid radiological research and day-to-day radiological work.

\section{RESULTS}

ET Net has been in successful operation for 3 years. Overall, the conferences devoted to computers in health sciences education have been popular, with participation consisting of several hundred ongoing discussions. The "digital_images" conference, which is devoted to computing in radiology, contains more than 50 ongoing discussions. Nonetheless, participation by radiologists has been somewhat slow in growing, probably because of the lack of publicity. It is hoped that, with further publicity, a critical mass of regularly participating radiologists will congregate on-line to fulfill the great promise that computer conferencing offers to radiology.

\section{DISCUSSION}

ET Net's advanced computer conferencing software allows individuals who are separated in space and time to unite electronically to participate, at their convenience, in interactive discussions on applications of computers in health sciences education and radiology. A computer conferencing system such as ET Net provides the valuable service of allowing health sciences professionals, eg, radiologists, to maintain regular contact with colleagues on issues when they are not physically together.

Computer conferencing software is a form of computer software that facilitates a structured and organized exchange of on-line information and ideas. It is a distinct improvement over the more widely known computer bulletin board software that facilitates an unstructured and unorganized exchange of on-line information and ideas. Reading a computerized bulletin board system is analogous to trying to make sense out of a cork bulletin board filled with post-it-notes. On the other hand, reading a computer conferencing system is analogous to reading a well-organized book, with each chapter being a separate item or discussion.

Although the benefits of telecommunication for the radiologist were documented long ago, ${ }^{1}$ only a few computer bulletin boards and conferencing systems exist that are dedicated to radiology. A MEDLINE search found only one article relating to computer bulletin board systems in the radiology literature. ${ }^{1}$ However, because computers are playing an ever more central role in our personal and professional lifes, more radiologists will become connected to computer networks such as the Internet. As this happens, their need for services such as those provided by ET Net will grow, and ET Net will be available for their professional use.

\section{ACKNOWLEDGMENT}

The authors thank Nola Riley for her secretarial assistance, Phyllis Bergman for her editorial assistance, and Daniel R. Masys, MD, for providing the support to allow the initial creation and subsequent operation of this project.

APPENDIX: HOW TO JOIN ET NET

One may follow the attached instructions to log on to ET Net for the first time. All one needs to access ET Net is a personal computer with communications software and a modem. Participation on ET Net is open to all health sciences professionals and students. Please note that, in the below instructions, when you are asked to type "something," do not type the surrounding quotation marks. Always hit $\langle$ RETURN $\rangle$ after typing a command. Please also note that these instructions are designed to help you log on to ET Net via SprintNet. If you wish to log on to ET Net via the Internet, skip to step 18.

1. First find the SprintNet node nearest to you. To do so, dial 1-800-877-5045 and press extension 5 (SprintNet Information). Tell the Sprint Net operator where you live and what speed your modem is. They will tell you the correct number to call. If you don't have a local SprintNet 
node, contact the Lister Hill National Center for Biomedical Communications Educational Technology Branch at the phone number given below for assistance.

2. Make sure your communications software is set to even parity, 7 data bits, 1 stop bit. Dial your local SprintNet node telephone number.

3. Note that you can communicate with SprintNet at either 1,200 or $2,400 \mathrm{bps}$. After dialing SprintNet and making a connection, if you are trying to connect at 1,200 bps, type $\langle$ RETURN $\rangle\langle$ RETURN $\rangle$. If you are trying to connect at 2,400 bps after SprintNet connects you, type ' $a$ ' $\langle$ RETURN $\rangle$. When SprintNet asks for "TERMINAL=", type 'D1' and hit $\langle$ RETURN $\rangle$.

4. When SprintNet gives you an " $a$ ” prompt, type 'C 301565 ' and hit $\langle$ RETURN〉.

5. ET Net will then display "etnet! Please enter network login:", and you should type 'etnet' and hit (RETURN).

6. Follow ET Net's prompts, and when it asks you to enter your last name, type 'newuser' $\langle$ RETURN $\rangle$. Please be sure to type 'newuser' in lower case.

7. Now ET Net will take you through a registration process. Please enter a seven-character user ID consisting of the first seven letters of your last name. From now on, ET Net will prompt you for this seven-character user ID when you log on (this is what ET Net wants when it prompts you for your "last name"), so make sure you write it down and keep it in a safe place along with your password. If someone else with the same last name as you has joined ET Net before you, when you type your last name in for the first time, ET Net will tell you "that name is already in use" and prompt you to pick another last name. At this point, enter a different seven-letter long combination of characters that you will easily remember. For example if your name is "John A. Smith" and you entered "jasmith" as your last name and ET Net told you "that name is already in use," you could enter "josmith" or "smithja" or "smithxx," etc, as your last name. If you are having problems logging on to ET Net for the first time, don't hesitate to give the Lister Hill National Center for Biomedical Communications Educational Technology Branch a call at the phone number listed below for help.

8. During the registration process, you will be asked to enter your complete name, telephone number, and a brief introduction. Please provide all the information requested and be sure to include your mailing address and electronic mail address in your brief introduction, so that others can contact you personally via phone, letter, or electronic mail.

9. When you have completed the registration process, ET Net will ask you to "JOIN which conference?", and, at this point, you should type 'users_guide' and hit 〈RETURN〉. You will then be asked "Do you wish to join this conference?" Type 'yes' and hit (RETURN). Note that ET Net is set up by default to pause after it types out 23 lines of output. To continue, always hit $\langle$ RETURN $\rangle$.

10. ET Net is set up to work by default with menus, which is time consuming. At this point, issue the following commands to turn the menus off. From the Main Menu, type a ' 7 ' (User Environment) and hit (RETURN $\rangle$. Then, at the User Environment Menu, type a ' 3 ' (Change My Interface) and hit (RETURN). Finally, from the Interface Style Menu, type a '5' (Use Caucus Command Line) and hit (RETURN). Menus are now turned off, and the rest of this guide assumes that they are. If you ever want to turn menus back on, type 'MENU' from an "AND NOW?" prompt.

11. You are inside the users _ guide conference, which will teach you the basic commands you need to know to use ET Net. Type 'show all items pass' and hit (RETURN〉 and you will be given an introduction on how to use ET Net. If you wish, you can save it and print it out for future reference.

12. After finishing the users_guide conference, from an "AND NOW?" prompt, type "join digital_images' to join the digital_images conference. You will be asked by ET Net "Do you wish to join this conference?" and you should type 'yes' and hit $\langle$ RETURN $\rangle$. Once inside the digital images conference, from an "AND NOW?" prompt, type 'show all items' and you will be shown 
each item in turn, allowing you to add responses. Here, you will be able to participate in discussions relating to applications of computers in radiology.

13. Remember that anytime you are confused by ET Net, just type 'help' and hit (RETURN〉 to get assistance.

14. When you are finished using ET Net, type 'bye' to log off.

15. If you are calling from Canada, you first must get the phone number of your local DATAPAC node. First try looking in your local telephone directory. Next, you can call the DATAPAC Information Center at 1-800-267-6574. Finally, if all else fails, contact the Lister Hill National Center for Biomedical Communications Educational Technology Branch. After dialing the local DATAPAC node and making the connection, type 3 periods '.. ' Then when DATAPAC prompts you, type the following route code: '1 311030100565 ' and then hit $\langle$ RETURN $\rangle$. Then continue on starting at step 5.

16. If you are trying to connect to ET Net from a country other than the USA or Canada, please contact ET Net in writing at the address given in care of Dr Susan Sparks to obtain instructions on how to connect to ET Net from your country.

17. If you are on the Merit Computer Network, you can connect to ET Net by entering 'etnet' at a "Which Host?" prompt. Then continue on starting at step 5.

18. If you are on the Internet, you can connect to ET Net by using the telnet command to connect to the Internet host 'etnet.nlm.nih.gov' and then continue on starting at step 5.

For further help contact: ET Net Coordinator, Educational Technology Branch, Lister Hill National Center for Biomedical Communications, National Library of Medicine, 8600 Rockville Pike, Bethesda, MD 20894. Phone: (301) 496-0508; FAX: (301) 480-3035. E-mail: tlc@lhc.nlm.nih.gov

\section{REFERENCE}

1. Rowberg AH. Newell JD, Hunter TB: Telecomputing in radiology. Am J Roentgenol AJR 145:411-413, 1985

\section{ERRATA}

Addendum to Datz F, Rosenberg C, Gabor $F$, et al: The use of computer-assisted diagnosis in cardiac perfusion nuclear medicine studies: A review (part 3). J Digit Imaging 6:67-80, 1993

Charles Rosenberg, PhD, is a GRECC Investigator at the Department of Veterans Affairs Geriatric Research, Education and Clinical Center, VAMC SLC UT. Portions of the material presented in that article were based on work supported by the Department of Veterans Affairs Geriatric Research, Education and Clinical Center, Salt Lake City, UT. 